\title{
Selecting and maintaining a diverse T-cell repertoire
}

Ananda W. Goldrath \& Michael J. Bevan

Department of Immunology and Howard Hughes Medical Institute, University of Washington, Seattle, Washington 98195, USA

To provide a T-cell population that will respond promptly to foreign antigen, the immune system looks inward, using the variety of self-antigens to select and maintain a diverse repertoire of receptors. A protective immune system must include a T-lymphocyte population that is poised to respond to foreign antigenic peptides presented by self-major histocompatibility complex molecules. As the organism cannot predict the precise pathogen-derived antigens that will be encountered, the system uses the diverse array of self-peptides bound to self-major histocompatibility complex molecules, not only to select a receptor repertoire in the thymus, but also to keep naïve T cells alive and 'ready for action' in the periphery.

The revelation that $\mathrm{T}$ lymphocytes recognize foreign antigen only when presented by major histocompatibility complex (MHC) molecules was made 25 years ago and remains the cornerstone of our understanding of cellular immunity ${ }^{1}$. The nature of antigen presentation was unknown for more than a decade until it became clear that foreign antigen has to be processed into peptides that can bind stably to the groove of an MHC molecule to allow recognition by the T-cell receptor $(\mathrm{TCR})^{2,3}$. MHC genes are extremely polymorphic and the various allelic products bind a broad spectrum of different peptides, explaining MHC restriction of T-cell recognition. During T-cell development, immature $\mathrm{T}$ cells are chosen to mature on the basis of the ability of their clonally expressed TCR to interact weakly with self-MHC molecules expressed in the thymus. During this process of positive selection, self-peptides bound in the MHC groove are also recognized. Thus, the selection of the TCR repertoire is not based solely on the recognition of one or a few self-MHC structures, but on the recognition of those structures modified by the binding of an enormous diversity of self-peptides. Low-affinity interactions between the newly expressed TCRs and self-peptideMHC molecules select a diverse repertoire that will be fine-tuned to react strongly to pathogen-derived peptides bound to the same $\mathrm{MHC}$ in the periphery. Even after maturing and leaving the thymus, $\mathrm{T}$ cells continue to depend on survival signals transmitted by the interaction of the TCR with self-peptide-MHC complexes. The long-term homeostasis of naïve $\mathrm{T}$ cells depends on continuous tickling of the TCR, and the rebound of the size and complexity of the peripheral lymphocyte pool after any marked loss in cell numbers involves recognition of self-peptide-MHC complexes. Infection often results in massive clonal expansion and development of effector function that initially overrides the balance of lymphocyte pools. When the pathogen is cleared, balance is restored: effector cells are eliminated and a long veteran's retirement is guaranteed for the surviving memory cells, which are no longer dependent on recognition of self-antigen. The veterans may be recalled to action quickly by a subsequent infection with the same pathogen. To provide protection against both unknown new pathogens and the recurrence of old infections, the immune system must maintain separate populations of naïve cells and memory cells. In addition, the system must retain an appropriate mixture of $\mathrm{CD}^{+}$helper T cells and $\mathrm{CD} 8^{+}$cytotoxic $\mathrm{T}$ cells in both naïve and memory pools.

\section{Thymocyte development}

Bone marrow stem cells enter the thymus and commit to the $\mathrm{T}$ lineage in response to signals from the microenvironment ${ }^{4}$. The stages of development of the major lineages of $\mathrm{T}$ cells are delineated by the surface expression of the co-receptor molecules CD4 and
CD8. The earliest precursors are $\mathrm{CD} 4^{-} \mathrm{CD} 8^{-}$double negative (DN) in phenotype, comprising about $3 \%$ of the total thymocyte numbers. These proliferate extensively and become $\mathrm{CD} 4^{+} \mathrm{CD} 8^{+}$double positive (DP) cells, which are still immature and are the targets for TCR-based selection events. The MHC molecules expressed by the epithelial elements of the thymus are critically involved in the process of positive selection, which allows a small fraction of the DP population to mature into single positive CD4 lineage or CD8 lineage $\mathrm{T}$ cells ${ }^{5,6}$.

\section{Assembling the TCR}

As is the case for antibodies, functional TCR genes are assembled from separate $\mathrm{V}, \mathrm{D}$ and $\mathrm{J}$ region segments by genetic recombination ${ }^{7}$. For $\mathrm{T}$ cells expressing $\alpha \beta$ receptors, this process of gene rearrangement occurs in the thymus. Mice and humans carry in their germline a large number of $\mathrm{V}$ gene segments, which encode roughly the first 90 residues of a mature TCR $\alpha$ or TCR $\beta$ chain. The TCR $\beta$ chain gene is assembled at the DN stage of T-cell development. A short D gene segment is juxtaposed to a short J segment and this is followed by rearrangement of a $\mathrm{V}$ segment to the assembled downstream DJ segment. In the case of TCR $\alpha$ chains which rearrange later at the DP stage there are no D segments; a large number of $\mathrm{V} \alpha$ segments may rearrange to a large number of $\mathrm{J}$ segments. The immunoglobulin-like fold of the TCR chains positions three loops or complementarity determining regions (CDRs) from each chain in close proximity to each other, creating a binding face that will contact antigen. Two of these CDR loops, namely 1 and 2 , are encoded by the $\mathrm{V}$ gene segment and have only the diversity provided by the number of germline $\mathrm{V}$ region gene segments, around $20-70 \mathrm{~V} \alpha$ and $\mathrm{V} \beta$ segments in mice and humans. The third CDR loop is created by the juxtaposition of VJ or V(D)J segments and provides much more diversity, a result of the ability of each $\mathrm{V}$ segment to rearrange to any (D)J segment compounded by the fact that the joining of the coding sequences is imprecise. Nucleotides may be added or deleted at each of these junctions. If there were only $50 \mathrm{~V} \alpha$ and $50 \mathrm{~V} \beta$ genes to encode the TCR repertoire, combinatorial pairing would provide only $50 \times 50=2,500$ TCRs. The addition of (D)J segments and the imprecision of joining boosts this number into the billions, with sequence diversity concentrated in the CDR3 loops.

\section{TCR interaction with ligand}

Crystal structures of MHC class I and class II molecules have revealed their mode of function (Fig. 1). Although differing in detail, both MHC class I and class II molecules have peptidebinding grooves to present antigen to $\mathrm{T}$ cells ${ }^{8-10}$. In each case, the groove is made up of a 'floor' of $\beta$-sheets and 'walls' of long 
a

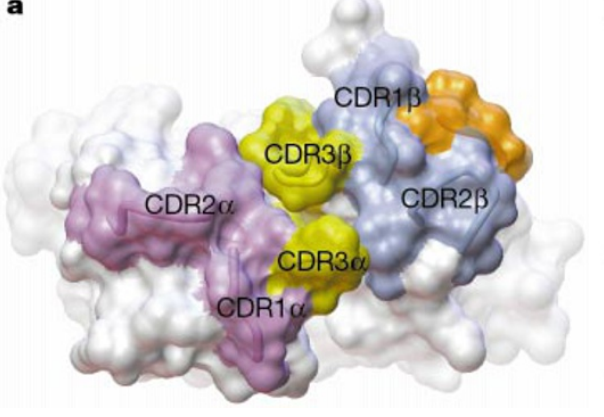

b

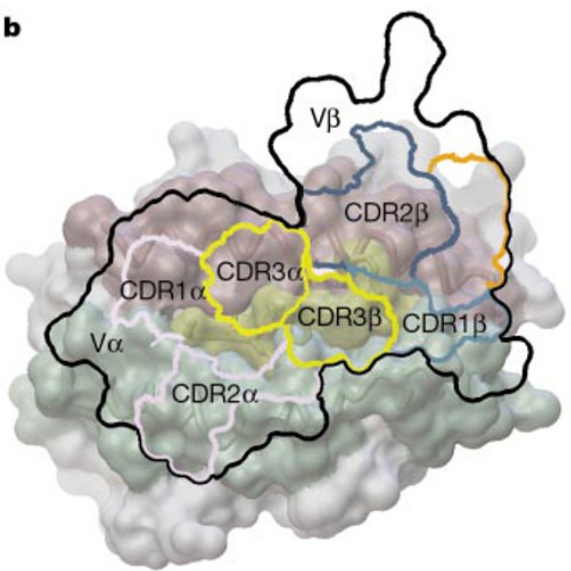

c

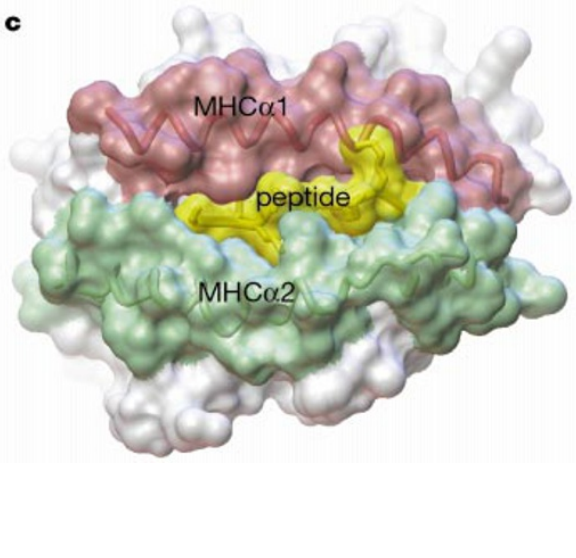

Figure 1 The nature of TCR interaction with peptide-MHC ligand. a, Positioning of the three CDR loops of the TCR $\alpha$ and TCR $\beta$ chains in the ligand-binding face of the TCR. b, Footprint of the TCR on the peptide-MHC complex. c, MHC class I molecule with bound peptide. The long $\alpha$-helices of MHC enclose the peptide. Reprinted with permission from Garcia et al. ${ }^{11}$. The crystal structure of a TCR interaction with a MHC class II ligand has not been reported but is likely to follow the same rules. $\alpha$-helical regions. The most polymorphic residues in the MHC molecules protrude into the groove, thus altering the spectrum of peptides that can be bound. The remarkable feature of MHCpeptide binding is that it is both extremely stable yet promiscuous, allowing many different peptides to bind each allele. Many of the stabilizing interactions are between the backbone of the peptide and the MHC, whereas a few pockets in the groove select for certain side chains on the so-called 'anchor residues' of the peptide. MHC class I molecules load peptides soon after synthesis in the endoplasmic reticulum before trafficking to the cell surface. In contrast, the groove of MHC class II molecules is protected from peptide loading until the molecules reach endocytic vesicles. Once loaded with a peptide derived from lysosomal processing, the class-II-peptide complex, like the class-I-peptide complex, is expressed stably at the cell surface.

Mature T cells discriminate between self and foreign by recognition of peptides presented in the groove of self-MHC molecules. The membrane-distal face of the peptide-MHC complex that is presented to the $\mathrm{T}$ cell consists of a flat surface made up principally of the $\alpha$-helices of the MHC surrounding a central peptide. Highresolution structures of the TCR complexed with peptide-MHC ligand have shown how this is achieved (Fig. 1). Crystal structures of a murine TCR interacting with MHC class I and peptide ${ }^{11,12}$, and two human TCRs complexed with the HLA-A2 class I molecule bound to the same viral peptide $\mathrm{e}^{13,14}$ have been solved, and all of the structures showed the same basic topology of interaction ${ }^{11-16}$. The TCR is positioned in a diagonal orientation over the face of the peptide-MHC complex with the CDR1 and CDR2 loops of the TCR $\alpha$ chain lying over the N-terminal half of the peptide and the corresponding regions of the TCR $\beta$ chain lying over the C-terminal half. Many of the interactions of the V gene segment-encoded CDR1 and CDR2 regions of the TCR are actually with MHC residues. In particular, the CDR1 and CDR2 loops of the $\mathrm{V} \alpha$ chain have almost identical sites of interactions with peptide-MHC in all structures solved so far, and this match-up may be critical in establishing the overall configuration. The most variable loops of the TCR, namely the CDR3 regions of the TCR $\alpha$ and TCR $\beta$ chains, lie centrally and have most contact with protruding peptide side chains. Thus, the most variable region of the TCR (CDR3 loops) has best access to the most variable region of the ligand (peptide).

\section{The unselected TCR repertoire}

The lymphoid-specific components of immunoreceptor gene rearrangement, the recombinase-activating gene (RAG) products, are first expressed in thymocytes soon after entry in the thymus and operate initially on the TCR $\beta$ loci. Successful VDJ rearrangement resulting in the production of a functional, in-frame TCR $\beta$ chain is tested by assembly of the new gene product (TCR $\beta$ ) along with an invariant surrogate TCR $\alpha$ chain, pre-T $\alpha$, plus signalling components of the TCR, the CD3 complex ${ }^{17}$. Proper assembly of this complex with the newly generated TCR $\beta$ chain signals the CD4 $\mathrm{CD} 8^{-}$pre-T-cell to switch off expression of RAG proteins, to go through several rounds of division and to proceed to the $\mathrm{CD} 4^{+} \mathrm{CD} 8^{+}$ stage of thymocyte maturation. This check point, called $\beta$-selection, does not involve the recognition of any ligand by the pre-TCR, as truncated versions of TCR $\beta$ and pre-T $\alpha$ lacking the extracellular domain can trigger progression to the DP compartment ${ }^{18}$. The mere assembly of the CD3-pre-T $\alpha$-TCR $\beta$ complex appears to be sufficient to activate the downstream signalling pathway. It is possible, although perhaps unlikely, that some TCR $\beta$ chains are lost in this selection if they cannot assemble with CD3 and/or pre-T $\alpha$.

At the DP stage of thymocyte differentiation, the RAG genes are expressed once again, and the cells now focus on rearranging TCR $V \alpha$. to J $\alpha$. Production of a new TCR $\alpha$ chain that can pair with the existing TCR $\beta$ chain allows surface expression of the TCR $\alpha \beta-$ CD3 complex. Co-expression of randomly rearranged TCR $\alpha \beta$ at the DP cell surface is an expression of the germline, or unselected TCR repertoire. The repertoire that is allowed to leave the thymus for the peripheral lymphoid organs is only a subset of this larger pool. To complete maturation, the germline repertoire is positively and negatively selected for interactions with MHC molecules in the thymus. Ultimately, only about $5 \%$ of the DPs are allowed to emigrate. In a murine thymus that lacks expression of any $\mathrm{MHC}$ molecule, T-cell development is blocked at the DP stage. DP thymocytes from such a MHC-deficient environment are immature and unresponsive to conventional antigen, yet tricks have been used to study the specificity of their germline TCRs. In one case, maturation of the thymocytes was induced in foetal thymic organ culture by receptor engagement with anti-TCR antibodies ${ }^{19}$. The mature $\mathrm{CD} 4{ }^{+} \mathrm{T}$ cells that developed were cloned as hybridomas to allow analysis of their TCR specificity. In another case, DP thymocytes from MHC-deficient mice were isolated and reaggregated with MHC wild-type thymus stromal cells. In such reaggregate cultures, the fraction of cells that respond to MHC class I or MHC class II could be scored by examining the upregulation of surface markers that are normally turned on during thymocyte selection ${ }^{20}$. Both of these approaches showed that the germline TCR repertoire is skewed to the recognition of MHC molecules. Somewhere between 5 and $20 \%$ of the DP population from MHC-deficient mice was responsive to the set of $\mathrm{MHC}$ molecules expressed in any one mouse 


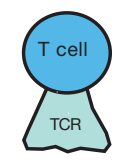

No interaction

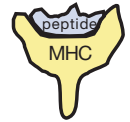

Thymocytes

Death by neglect

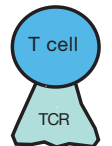

Low affinity

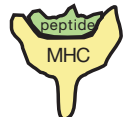

Positive selection: survival and maturation death

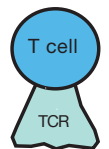

High affinity

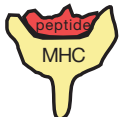

Negative selection
Peripheral T cells

Poor survival and inefficient division
Survival and division
Rapid proliferation: and memory cells generation of effector

Figure 2 Consequences of TCR interactions with ligands of varying affinity in the thymus and periphery. TCR-MHC interactions may facilitate maturation, survival, proliferation or death. The consequences of TCR binding to peptide-MHC are dictated by the affinity of the interaction, as well as the maturation state of the T cell. Immature thymocytes require a positively selecting signal, which is delivered through low-affinity interactions of their newly rearranged TCR with self-peptide-MHC. If no signal is delivered to the thymocyte because of an inability of the TCR to interact with self-peptide-MHC complexes, it will undergo programmed cell death known as death by neglect. Alternatively, a thymocyte may express a TCR that has high affinity for self-peptide-MHC. These cells must be eliminated by negative selection as they are potentially autoreactive. In the periphery mature T cells require low-affinity interactions with self-peptide-MHC to ensure survival. Furthermore, low-affinity interactions with self-peptide-MHC allow naïve $\mathrm{T}$ cells to expand when T-cell numbers are reduced. High-affinity interactions between a naïve Tcell in the periphery and peptide-MHC typically initiate the immune response against a foreign antigen. This leads to rapid proliferation and the development of the effector function culminating in the emergence of a few antigen-experienced cells that join the pool of memory $T$ cells to await a subsequent encounter with antigen.

strain. This is a much higher frequency than would be expected by the generation of receptors through the random rearrangement of gene sequences that do not have an inherent predisposition for interactions with MHC. Evolution has shaped the TCR genes, presumably the CDR1 and CDR2 regions encoded by the $\mathrm{V}$ gene segments, so that their $\alpha \beta$ product will preferentially match MHC molecules.

Certain V $\alpha$ gene segments have evolved to interact preferentially with either class I or class II molecules. TCRs bearing these $\mathrm{V} \alpha$ segments are preferentially selected into the $\mathrm{CD} 8^{+}$(MHC class I reactive) or $\mathrm{CD}^{+}$(MHC class II reactive) T-cell subset during normal thymocyte selection. Swapping individual residues in the $\mathrm{CDR} 1$ or CDR2 regions of the V $\alpha$ gene is sufficient to change the selection of these TCRs from the $\mathrm{CD}^{+}$to the $\mathrm{CD}^{+}$subset $^{21}$. Furthermore, certain mouse strains differ consistently in the ratio of $\mathrm{CD} 4^{+}$to $\mathrm{CD} 8^{+} \mathrm{T}$ cells in peripheral organs, and one of the loci controlling this phenotype maps with the TCR $\alpha$ locus $^{22}$.

\section{Positive selection of thymocytes}

Even though they express a TCR at the surface, in the absence of $\mathrm{MHC}$ recognition $\mathrm{CD} 4^{+} \mathrm{CD} 8^{+}$thymocytes will undergo apoptotic death because of receptor neglect (Fig. 2). During their approximate 3-day life span, the DP thymocytes will continue to express the RAG genes and continue to rearrange TCR $\alpha$ chain genes. TCR engagement with MHC class I or class II molecules on the cortical thymic epithelial cells is required to rescue the cell from death by neglect and to switch off further TCR $\alpha$ chain gene rearrangement. Only those DP thymocytes that bind self-MHC with sufficiently low affinity will be rescued. In contrast, DP cells that express a receptor that interacts with high affinity with MHC in the thymus will be eliminated in a process referred to as negative selection. Almost half of the cells reacting with self-MHC are lost through this mechanism $^{23}$ (Fig. 2). The low-affinity recognition of self-MHC not only promotes thymocyte survival but also determines commitment of the DP thymocyte to either the CD4 or the CD8 lineage ${ }^{5,6,24}$. Recognition of MHC class I results in commitment to the CD8 lineage, whereas recognition of class II results in $\mathrm{CD} 4^{+} \mathrm{T}$ cells. Thus, function and lineage are correlated with $\mathrm{MHC}$ class recognition. How positive selection on MHC class I versus class II is distinguished by the thymocyte is unknown. Lineage choice may be steered by qualitative or quantitative differences in signalling, or may be random but confirmed by later selective events.

The molecular nature of the TCR-MHC recognition event in positive selection has been an area of active research. Initial experiments showed that mutations affecting only the inside of the groove of a murine class I molecule drastically altered the selected repertoire of $\mathrm{CD}^{+} \mathrm{T}$ cells ${ }^{25,26}$. These experiments suggested strongly that self-peptides bound to the MHC groove are recognized by thymocytes and are critical for positive selection. Further analysis of this recognition required the use of an in vitro organ culture system, using thymus lobes from mice deficient in class I expression in which peptides could be added back to reconstitute positive selection. Disruption of the $\beta_{2}$-microglobulin gene, encoding the light chain of class I molecules, or of the TAP genes encoding a transporter that delivers peptides to nascent class I molecules in the endoplasmic reticulum, leads to a drastic decrease in selection of CD8 lineage cells. Surface expression of MHC class I can be restored to some extent by the addition of single peptides or peptide mixtures to organ culture systems. Initial experiments using thymic lobes from mice that express a normal, germline TCR repertoire suggested that a complex mixture of peptides restored selection of $\mathrm{CD}^{+} \mathrm{T}$ cells better than single peptides which bound and stabilized MHC to the same degree ${ }^{27,28}$. Follow-up experiments in this peptide add-back system using mice that express rearranged TCR transgenes of known ligand specificity suggested a stringent requirement for peptide recognition in the positive selection of CD8 lineage $T$ cells $^{29-31}$. Peptides that bind MHC class I and form a lowaffinity ligand for the TCR induced the maturation of functional $\mathrm{CD}^{+}$cytotoxic $\mathrm{T}$ cells most efficiently ${ }^{32}$. The measured TCR affinities for foreign peptide-MHC ligands fall into the $1-10 \mu \mathrm{M}$ range $^{33}$. Compared with this, the affinities for ligands that can efficiently induce positive selection, but cannot induce negative selection, are only 5-9-fold lower ${ }^{34}$.

Is a single self-peptide actually responsible for the maturation of a cohort of thymocytes? Or do a larger number of self-peptides select each TCR? Natural peptides eluted from MHC class I molecules can induce selection of certain TCR transgenic thymocytes when added back to TAP-deficient thymus lobes ${ }^{35,36}$, although whether these are expressed at the appropriate level in the thymus cortex is not known. Antibodies that react in a peptide-specific fashion with only a subset of class II molecules prevent the thymic selection of a specific subset of $\mathrm{CD}^{+}{ }^{+} \mathrm{T}$ cells ${ }^{37}$. Certainly, it can be concluded that a subset of self-peptides, and possibly a single self-peptide, bound to self-MHC acts as the ligand that permits the maturation of each $\mathrm{T}$ cell. In this way, these self-peptides act as low-affinity mimics or 'stand ins' to prime the immune system to interact later in the periphery with high-affinity ligands for the TCR such as foreign peptide bound to the same $\mathrm{MHC}^{38}$.

In the study of $\mathrm{CD} 4^{+} \mathrm{T}$-cell selection in the thymus, researchers have generated mice in which all or a large fraction of the class II molecules in an individual mouse are occupied by the same peptide, and have asked how many $\mathrm{T}$ cells can one peptide-MHC ligand select. The invariant chain Ii is a chaperone for MHC class II that contains a region, CLIP, that occupies the class II groove in the 
endoplasmic reticulum and prohibits other peptides from binding until the molecules reach endosomal/lysosomal vesicles. At this site, CLIP is removed by the action of the peptide exchange catalyst, $\mathrm{H} 2-$ M. Mice deficient in H2-M express near normal levels of class-IIpeptide on their surface, but in this case, most remain occupied by the CLIP peptide ${ }^{39-41}$. Another strategy has been to generate a covalently linked class-II-peptide construct that assembles with the single peptide 'sewn in' to the MHC groove and to express these as transgenes in mice that lack wild-type MHC class $\mathrm{II}^{42,43}$.

Initial results from these 'single peptide' systems were remarkable in showing that the mice developed a large and broad $\mathrm{CD} 4^{+} \mathrm{T}$-cell repertoire. They contained $20-50 \%$ of the normal numbers of $\mathrm{CD} 4^{+}$ $\mathrm{T}$ cells, with diverse TCRs as assessed by V $\beta$ and $\mathrm{V} \alpha$ usage. Could a single peptide-MHC complex select a near-normal $\mathrm{CD} 4^{+} \mathrm{T}$-cell repertoire? More recent analysis has shown quite clearly that the repertoire in these animals is far from normal. First, none of the single-peptide mice can positively select TCRs of known specificity that are selected in normal mice ${ }^{44-46}$. Second, closer analysis of the TCR $\alpha$ chain usage in the mutant versus wild-type mice shows that the selected repertoire is markedly constrained in the mutants ${ }^{47}$. Last, even when $95 \%$ of class II molecules are occupied by a single peptide, most of the $\mathrm{CD} 4^{+} \mathrm{T}$ cells that emerge from the thymus are actually selected on the $5 \%$ of molecules that are occupied by a diverse mixture of unknown self-peptides. The CLIP region of Ii was replaced with a tight-binding peptide, so that in mice that were Iideficient, $95 \%$ of surface MHC class II molecules were occupied by the substituted sequence ${ }^{48}$. Under these conditions, large numbers of $\mathrm{CD} 4^{+} \mathrm{T}$ cells were selected. The critical experiment was to breed these mice to an $\mathrm{H} 2-\mathrm{M}$-deficient background which resulted in no apparent change in the level of MHC class II expression, but a $70 \%$ decline in the number of $\mathrm{CD}^{+} \mathrm{T}$ cells selected. The simple conclusion is that the 'background' of diverse peptides bound to class II must have a major role in selection even though such complexes are present at extremely low levels.

Positive selection of immature DP thymocytes by low-affinity interactions with self-peptide-MHC complexes implies that cells at this stage of development are more sensitive to low-affinity ligands than are their mature descendents. This has been shown to be the case, despite the fact that they express 10 -fold less TCR on their surface $^{49,50}$. Low-affinity ligands stimulated early activation events in DP thymocytes but not in mature T cells bearing the same TCR; however, from what we have learned recently about the homeostasis of peripheral $\mathrm{T}$ cells, we might assume that under certain circumstances, mature $\mathrm{T}$ cells can also respond to low-affinity self-ligands.

\section{Peripheral T-cell homeostasis}

After the selection and maturation of $\mathrm{T}$ cells in the thymus, naive $\mathrm{T}$ cells enter the periphery where they circulate throughout the secondary lymphoid organs awaiting an encounter with antigen. The organization of the many cell types that make up the immune system requires complex homeostatic mechanisms to assure the production and survival of each cell type in appropriate numbers to maintain proper immune function. One can imagine many different mechanisms by which appropriate homeostasis of lymphocyte populations is maintained, including control of the rate of production, division of mature cells, trafficking and cell death.

To maintain homeostasis of lymphocyte populations, the different cell types must be grouped and counted. Peripheral B- and T-cell populations appear to be independently regulated ${ }^{51}$. This conclusion is based on the fact that similar numbers of mature B cells are found in the periphery of normal mice and in mice that lack $\mathrm{T}$ cells ${ }^{52}$. Likewise, the number of $\mathrm{T}$ cells found in normal mice does not increase in B-cell-deficient mice $^{53}$. In addition, when small numbers of $\mathrm{T}$ cells are introduced into depleted hosts, they undergo expansion equivalently in mice deficient in both $\mathrm{B}$ and $\mathrm{T}$ cells and in mice deficient only in $\mathrm{T}$ cell $\mathrm{s}^{54}$. All these results suggest the independent homeostatic control of T-cell and B-cell numbers.
The pool of peripheral $\mathrm{T}$ cells consists of naïve $\mathrm{T}$ cells and previously activated antigen-experienced memory cells. Interestingly, naïve and memory T cells appear to occupy separate homeostatic niches. After reconstitution of T-cell-deficient mice with memory cells, the transferred cells expand to equal the same total number of memory cells in normal mice. Furthermore, it is not possible to increase the size of the memory pool by transferring large numbers of memory $\mathrm{T}$ cells, indicating that there is a set point for memory T-cell number even when no naïve T cells are present ${ }^{55}$. The separate regulation of memory and naïve pools makes teleological sense as it is vital to retain both a diverse naïve repertoire that can respond to invasion by yet unknown pathogens and a memory pool that can respond rapidly to previously encountered pathogens. If these two populations were in competition for survival, the diverse repertoire of naïve $\mathrm{T}$ cells could be replaced by rapidly expanding memory $\mathrm{T}$ cells or, conversely, new thymic emigrants could displace memory $\mathrm{T}$ cells resulting in the loss of immunological recall ${ }^{51}$.

Although $\mathrm{CD}^{+}$and $\mathrm{CD}^{+}$cells serve different functions in the immune system and recognize different MHC molecules, many results suggest that they occupy overlapping homeostatic niches ${ }^{51}$. First, relatively normal numbers of total $\mathrm{T}$ cells are found in mice deficient in MHC class I or II or in the CD8 or CD4 co-receptors, and thus lacking one T-cell subset. Second, small numbers of mature peripheral $\mathrm{T}$ cells will expand when introduced into a $\mathrm{T}$ cell-deficient host, and the co-transfer of large numbers of either $\mathrm{CD}^{+}{ }^{+}$or $\mathrm{CD}^{+}$mature $\mathrm{T}$ cells can suppress this expansion ${ }^{56,57}$. Last, in TCR transgenic mice in which the T-cell population is skewed toward either the $\mathrm{CD}^{+}$or $\mathrm{CD}^{+}$subset, total T-cell numbers are typically near normal. Thus, $\mathrm{CD}^{+}$and $\mathrm{CD}^{+} \mathrm{T}$ cells appear to be counted together for purposes of maintaining the homeostasis of $\mathrm{T}$ cell numbers. However, some distinction is clearly made between $\mathrm{CD}^{+}$and $\mathrm{CD}^{+} \mathrm{T}$ cells, as the ratio of $\mathrm{CD}^{+}$to $\mathrm{CD}^{+} \mathrm{T}$ cells is tightly regulated in normal mice and will rapidly readjust after transfer of large numbers of either subset ${ }^{56}$.

The size of the naïve pool of T cells remains more or less constant in spite of the continuing contribution of recent thymic emigrants, the loss of $\mathrm{T}$ cells due to normal cell death, the expansion and waning of effector populations during immune responses, and the diversion of cells into the memory pool after an immune response. The size of the peripheral T-cell pool does not appear to influence the rate of thymocyte production or export of naïve $T$ cells, as changes in the number of peripheral $\mathrm{T}$ cells do not change the rate of thymocyte output ${ }^{58}$. These data suggest that homeostatic control over the size of the naïve peripheral pool is regulated by the loss of $\mathrm{T}$ cells in the periphery ${ }^{58}$. How recent thymic emigrants are incorporated into the peripheral pool is not entirely understood. Clearly, recent thymic emigrants must displace other naïve $\mathrm{T}$ cells to maintain homeostasis of the peripheral T-cell pool. Newly generated $\mathrm{T}$ cells could either randomly displace resident $\mathrm{T}$ cells in the periphery or selectively displace resident cells on the basis of a criterion such as age $e^{51,58,59}$. Even in the absence of thymic export, the naïve pool remains rather constant in size, indicating that the life span of naïve $\mathrm{T}$ cells is under homeostatic control ${ }^{59,60}$.

Relatively little is known about the mechanisms that determine life span, capacity for renewal and survival of naïve $\mathrm{T}$ cells in the periphery in the absence of antigen. Naïve T cells were considered to be quiescent 'spore-like' cells that persist indefinitely without antigenic stimulation ${ }^{61-65}$; however, evidence now suggests that naïve $T$ cells may require input signals to support their survival in the periphery. Expression of transcription factors such as lung Kruppel-like factor and NFAT4 are crucial for the maintenance of a resting, naïve population of $\mathrm{T}$ cells ${ }^{66,67}$. In addition, cytokine signals influence the survival, homing and activation of naïve $\mathrm{T}$ cells, which in turn alter the activation state and homeostasis of the naïve compartment ${ }^{68,69}$. The bcl-2 family of molecules, which are intimately involved in the survival of many cell types, are also involved in the maintenance of the naïve pool of $\mathrm{T}$ cells ${ }^{70}$. Now it 
appears that TCR interactions with self-MHC direct a signal required for maintenance of naïve $\mathrm{T}$ cells.

\section{MHC in peripheral maintenance}

Although self-peptide-MHC molecules are known to be crucial in the generation of a T-cell repertoire with a wide spectrum of specificities, only recently have data emerged that implicate TCR interaction with self-MHC in the survival and expansion of peripheral T cells (Fig. 2). Several groups have shown that MHC molecules are important in maintaining a peripheral pool of $\mathrm{T}$ cells ${ }^{71}$.

In many experimental systems, naïve $\mathrm{CD} 4^{+} \mathrm{T}$ cells demonstrate a requirement for MHC class II molecules for long-term survival ${ }^{72-75}$. $\mathrm{CD}^{+} \mathrm{T}$ cells were positively selected in MHC class-II-expressing thymuses and then assayed for survival in a periphery that was engineered to lack expression of the positively selecting MHC class II allele. In all cases, $\mathrm{CD} 4^{+} \mathrm{T}$ cells declined in numbers in the absence of self-MHC class II. One report has shown that expression of MHC class II on dendritic cells is sufficient to support $\mathrm{CD} 4^{+} \mathrm{T}$ cells in the periphery $^{74}$.

An important role for MHC class I in maintaining naïve $\mathrm{CD}^{+} \mathrm{T}$ cells has been demonstrated by the transfer of TCR transgenic CD ${ }^{+}$ $\mathrm{T}$ cells into MHC class-I-deficient hosts ${ }^{76,77}$. Both studies showed that the naïve $\mathrm{CD} 8^{+} \mathrm{T}$ cells fade away in hosts that lack peripheral expression of MHC class I. Furthermore, the MHC class I allele that positively selected the $\mathrm{T}$ cell in the thymus is strictly required for naïve T-cell survival ${ }^{76}$. The inability of $\mathrm{CD}^{+} \mathrm{T}$ cells to accumulate in mice expressing very low levels of MHC class I when grafted with a wild-type thymus further supports a role for TCR engagement by $\mathrm{MHC}$ in the periphery to permit survival of $\mathrm{T} \mathrm{cells}^{78}$. Although some differences were reported in these studies, the trend remains clear: the retention of both $\mathrm{CD}^{+}$and $\mathrm{CD} 4^{+} \mathrm{T}$ cells is enhanced when MHC class I and II, respectively, are expressed in the periphery.

\section{MHC in recovery of peripheral homeostasis}

The naïve T-cell pool consists mostly of resting cells compared with the memory pool in which cells turn over more rapidly ${ }^{63}$. Upon close examination, however, low levels of division are observed in naïve T-cell populations in the absence of conventional antigen stimulation $^{61,63}$. Furthermore, even without conventional antigen stimulation, naïve $\mathrm{T}$ cells have a great potential for division when transferred into hosts with low numbers of lymphocytes ${ }^{56,60,62,65,79-83}$. This proliferation of naïve cells after transfer into lymphopenic hosts has been speculated to involve cytokine release, the expression of environmental antigens or interactions with self-MHC molecules ${ }^{57,65,82,84-86}$. Recently, the molecular interactions that support the expansion of naïve $\mathrm{T}$ cells in lymphopenic environments have become of significant interest, and several groups have studied the function of MHC in this process. There is a clear consensus that both $\mathrm{CD}^{+}$and $\mathrm{CD}^{+} \mathrm{T}$ cells require expression of $\mathrm{MHC}$ ligands for efficient homeostatic expansion in lymphopenic hosts ${ }^{54,57,77,86-89}$.

When polyclonal or TCR transgenic monoclonal CD4 ${ }^{+}$T-cell populations are transferred into syngeneic hosts that have been $\mathrm{T}$ cell depleted, they divide without direct antigenic stimulation. If the $\mathrm{CD}^{+}{ }^{+}$cells are transferred into class II-deficient hosts or hosts expressing MHC class II other than the positively selecting class II molecule, however, little division is observed ${ }^{54,57,86,87}$. Similarly, both polyclonal and TCR transgenic $\mathrm{CD}^{+} \mathrm{T}$ cells expand in wild-type hosts expressing MHC class I, but minimally in hosts with low or no levels of MHC class I molecules ${ }^{77,88,89}$. Thus, both $\mathrm{CD}^{+}$and $\mathrm{CD}^{+} \mathrm{T}$ cells proliferate much more efficiently in a lymphopenic host expressing MHC class I or MHC class II molecules, respectively. These data show that $\mathrm{MHC}$ recognition contributes to division of naïve $\mathrm{T}$ cells responding to homeostatic signals.

What is the specificity of the TCR-MHC interaction that promotes homeostasis-driven expansion? Experiments comparing $\mathrm{CD}^{+} \mathrm{T}$ cells transferred into hosts with wild-type self-peptide-
MHC class II or hosts expressing MHC class II bound to a limited repertoire of self-peptides show that $\mathrm{CD}^{+}{ }^{+} \mathrm{T}$ cells undergo expansion efficiently only when transferred into a host with a selfpeptide-MHC class II repertoire from the background in which the $\mathrm{T}$ cells developed. Division of wild-type $\mathrm{CD}^{+} \mathrm{T}$ cells was observed in T-cell-depleted wild-type hosts but only minimally in $\mathrm{H} 2-\mathrm{M}$-deficient hosts. As expected, $\mathrm{CD}^{+}{ }^{+} \mathrm{T}$ cells that were positively selected in the $\mathrm{H} 2-\mathrm{M}$-deficient background were able to divide when transferred into T-cell-depleted $\mathrm{H}$-2M-deficient hosts $^{57,86}$. These data make a strong case that peptide-specific recognition of MHC class II complexes by T cells promotes division in T-cell-depleted environments ${ }^{57,86}$. Furthermore, they suggest that this recognition is specific for the same peptides that $\mathrm{T}$ cells previously recognized with low affinity during their positive selection in the thymus ${ }^{57,86}$.

$\mathrm{CD}^{+} \mathrm{T}$ cells also exhibit a requirement for specific recognition of peptide-MHC class I complexes to undergo homeostatic expansion. Proliferation of TCR transgenic $\mathrm{CD}^{+} \mathrm{T}$ cells in TAP-deficient hosts expressing low levels of MHC class I was restored by the addition of a single MHC class-I-binding peptide ${ }^{89}$. Recognition was peptide specific, as a low-affinity peptide known to positively select the TCR transgenic T cells promoted expansion, whereas a control peptide did not. Thus, the signal for homeostatic proliferation may be characterized by low-affinity TCR interactions with self-peptide-MHC complexes. This suggests that the specific recognition of self-peptide-MHC complexes drives homeostatic proliferation of peripheral $\mathrm{T}$ cells, and that this interaction has a specificity similar to the recognition of self-peptide-MHC during positive selection. It is possible that the positively selecting peptide(s) may not necessarily be expressed in the periphery, but that other ligands of similar affinity or avidity for the TCR function in this capacity (Fig. 2).

\section{The response to $T$-cell depletion}

To what extent does homeostasis-driven proliferation of peripheral $\mathrm{T}$ cells in a self-peptide-MHC-specific manner contribute to a useful immune system? One possibility is that it ensures the diversity of the peripheral $\mathrm{T}$-cell repertoire. It is appealing to consider that a requirement for peptide-specific recognition during homeostatic proliferation would reinforce the requirement for self-recognition established during positive selection for an individual clone to survive over time and contribute to the longlived pool of peripheral T cells. Furthermore, the ability of a naïve $\mathrm{T}$ cell to receive TCR-self-peptide-MHC signals may determine to what extent a given cell contributes to reconstitution after a decline in T-cell numbers. This could have important implications for peripheral homeostasis after a loss of peripheral $\mathrm{T}$ cells in adults, as the ability of the thymus to replenish a naïve $\mathrm{T}$-cell pool with diverse specificities may be insufficient to repopulate the periphery efficiently $^{84}$. The clinical relevancy of such a mechanism certainly exists for patients with T-cell immunodeficiencies resulting from infection (such as AIDS) or from therapeutic treatments (such as chemotherapy or irradiation).

Until recently, it was thought that naïve T cells did not recognize self-peptide-MHC ligands in the periphery. However, the existence of mechanisms for MHC-dependent homeostasis-driven proliferation and survival suggest that low-affinity interactions between the TCR of peripheral T cells and self-peptide-MHC complexes are involved in regulating homeostasis of peripheral T-cell populations. $\mathrm{T}$ cells proliferate in response to low-affinity self-ligands when T-cell numbers are reduced, which demonstrates that the sensitivity of the TCR or the response to TCR-mediated signals can be altered by global homeostatic signals. We can envisage at least three different mechanisms by which this could work (Fig. 3). Under normal conditions, T cells crowding through lymph nodes may be competing for ligands on presenting cells. Competition may be for MHC itself or for some other growth factor; lymphopenia would decrease 
the competition. Alternatively, T cells may 'count' their own numbers by sending inhibitory signals to each other, or by inhibiting the stimulatory properties of a presenting cell. Decreasing T-cell numbers would sensitize the $\mathrm{T}$ cell or activate the presenting cell (Fig. 3).

One implication of naïve T cells being induced to proliferate in Tcell-depleted environments is that what has previously been considered 'maintenance' is likely to be the sum of survival and proliferation. The decline of T cells in the absence of MHC may be partially due to their inability to undergo homeostatic proliferation. Thus, it is necessary at this point to re-evaluate our understanding of T-cell maintenance to accommodate a more complex regulation of $\mathrm{T}$-cell homeostasis.

\section{Maintenance of the memory T-cell pool}

Memory $\mathrm{T}$ cells often divide in the periphery long after antigen stimulation, even without evidence for the persistence of antigen. Furthermore, the rules for the persistence of memory $\mathrm{T}$ cells appear to be different from those that naïve T-cells obey. Recent studies have shown that $\mathrm{CD} 4^{+}$and $\mathrm{CD} 8^{+}$memory $\mathrm{T}$ cells have a much less stringent requirement for TCR-MHC interactions for survival and homeostatic proliferation compared with naïve $\mathrm{T}$ cells.

The survival of memory $\mathrm{CD} 8^{+} \mathrm{T}$ cells is much less dependent on MHC class I than that of naïve $\mathrm{CD}^{+} \mathrm{T}$ cells. Both polyclonal and TCR transgenic memory $\mathrm{CD}^{+} \mathrm{T}$ cells specific for lymphocytic choriomeningitis virus (LCMV) survive indefinitely in MHC-
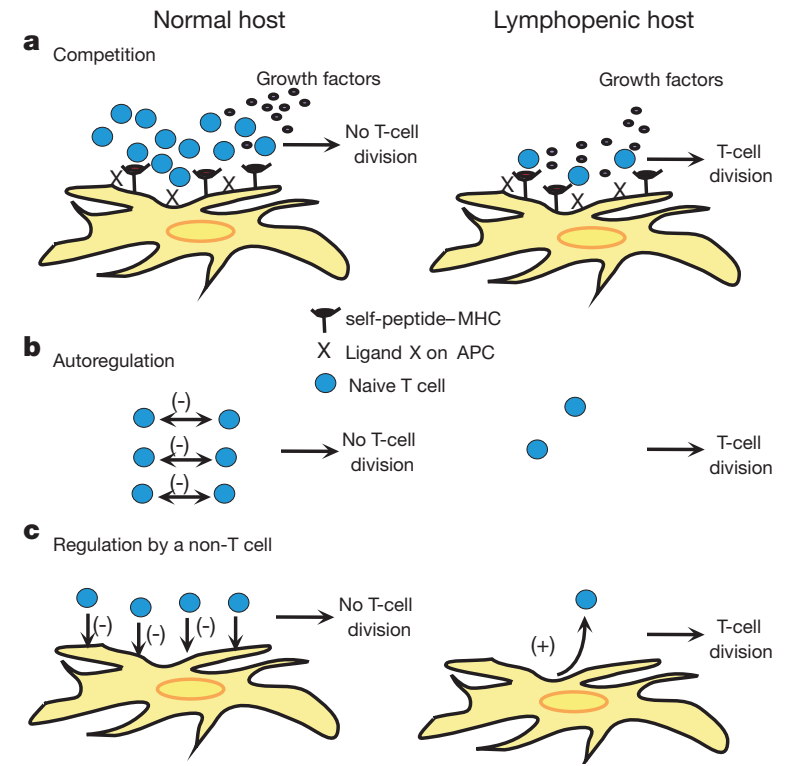

Figure 3 Possible mechanisms to explain the proliferation by naïve T cells in response to low-affinity ligands following T-cell depletion. The lymphopenic state renders naïve T cells capable of responding by proliferation to peptide-MHC complexes that are not stimulatory during normal conditions. a, Competition for niches or space on the antigen-presenting cell (APC) would be reduced when T-cell numbers are depleted, thereby allowing greater accessibility to ligands or growth factors. Such decreased competition for non-MHCderived stimulatory signals such as growth factors or ligands could make signals accessible that would allow T cells to detect self-peptide-MHC complexes resulting in proliferation. It seems improbable that a decrease in direct competition for peptide-MHC complexes explains the ability of $\mathrm{T}$ cells to proliferate in response to self-peptide-MHC ligands because $\mathrm{CD} 4^{+}$and $\mathrm{CD} 8^{+} \mathrm{T}$ cells recognize distinct ligands yet appear to occupy largely overlapping homeostatic niches. b, T cells could somehow 'sense' a drop in T-cell number. T cell/T cell interactions could inhibit proliferation or decrease sensitivity to TCR signals. A release from autoinhibition during lymphopenia would allow $T$ cells to receive a signal for proliferation following interactions with low-affinity ligands for the TCR. c, T-cell loss could be detected by another cell type that would upregulate the expression of a factor promoting T-cell division by influencing the T-cell response or sensitivity to TCR interactions with low-affinity peptide-MHC ligands. deficient hosts ${ }^{77}$. Memory CD8 ${ }^{+}$T cells, expressing the TCR transgene specific for the male antigen HY, survived and proliferated without a preference for the restricting allele ${ }^{76}$. However, unlike the LCMV-specific memory T cells, the memory $T$ cells specific for the $\mathrm{HY}$ antigen disappeared after transfer into mice expressing low levels of MHC class $\mathrm{I}^{76,90}$. In both experimental systems, CD8 ${ }^{+}$ memory $\mathrm{T}$ cells were able to proliferate in lymphopenic hosts even when MHC class I was not expressed, showing that homeostatic proliferation of memory $\mathrm{CD}^{+} \mathrm{T}$ cells is MHC class I independent ${ }^{76,77}$. Although some differences in conclusions from these studies may be attributed to distinct experimental systems, the data with polyclonal $\mathrm{CD}^{+} \mathrm{T}$ cells specific for many LCMV antigens present a convincing argument for MHC independence for homeostatic proliferation and survival of memory CD ${ }^{+} \mathrm{T}$ cells ${ }^{77}$. Similarly, memory $\mathrm{CD}^{+} \mathrm{T}$ cells survive indefinitely in hosts that do not express their positively selecting MHC class II molecule ${ }^{91}$ or any class II molecules at all ${ }^{92}$. Thus, $\mathrm{CD}^{+}$memory T cells appear to have no requirement for the presence of self-MHC class II to support their long-term survival. An obvious corollary of these observations is that neither $\mathrm{CD}^{+}$nor $\mathrm{CD}^{+}$memory $\mathrm{T}$ cells need continuous exposure to their cognate antigen to survive, a subject of much previous debate.

\section{The response to infection}

There is concern that certain infectious agents may overwhelm the immune system because of their high replicative potential; however, the opposite problem may arise. Analyses have shown that, in some cases, the specific immune response can threaten to overwhelm the lymphoid compartment. Use of peptide-MHC tetramers to stain T cells on the basis of TCR specificty, and of single-cell assays to detect interferon- $\gamma$ secretion, have revolutionized our appreciation of the numerology of the $\mathrm{CD}^{+}{ }^{+} \mathrm{T}$-cell response to infection ${ }^{93,94}$. For mice infected with the natural pathogen LCMV, total $\mathrm{CD}^{+}$T-cell numbers increase 2-10-fold by eight days after exposure (Fig. 4). Remarkably, most of the $\mathrm{CD}^{+}{ }^{+} \mathrm{T}$-cell expansion is antigen-specific! The rare, antigen-specific, naïve $\mathrm{CD}^{+} \mathrm{T}$ cells present before infection can divide $13-16$ times in this time, so that their frequency may go from 1 in $10^{4}-10^{5}$ to making up most of the $\mathrm{CD}^{+}$T-cell population. Such rapid proliferation leads to the production of

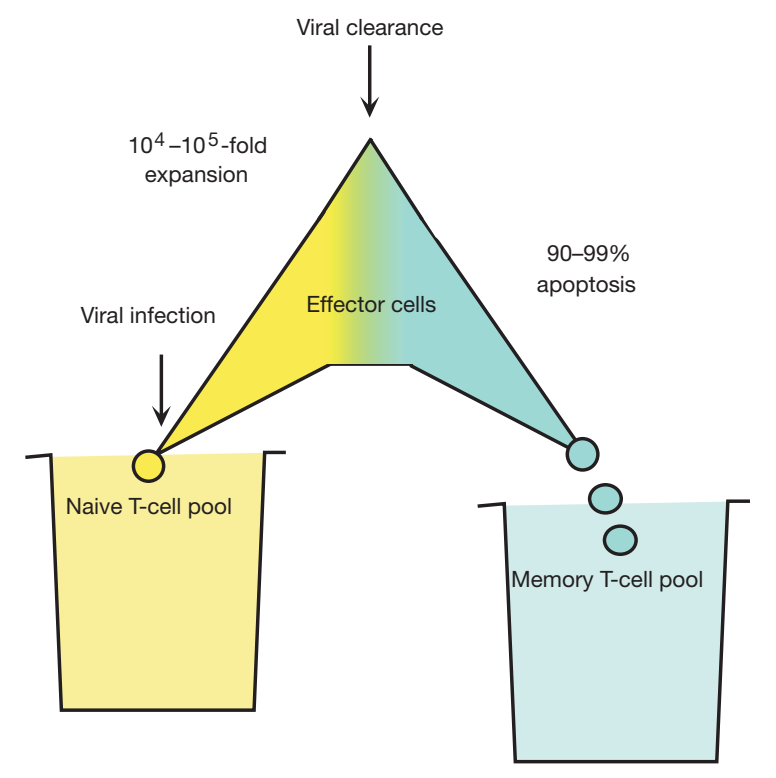

Figure 4 Response to infection and the recovery of homeostasis. The case of the murine $\mathrm{CD}^{+} \mathrm{T}$-cell response to $\mathrm{LCMV}$ is depicted. After viral infection, naïve $\mathrm{CD}^{+} \mathrm{T}$ cells that recognize LCMV epitopes may go through 13 or more divisions within 8 days, producing massive numbers of effector cells. When antigen is cleared, most of the effector cells die, leaving an expanded population of LCMV-specific T cells, which join the memory pool. 
enormous numbers of effectors capable of killing infected cells and releasing cytokines upon antigen encounter. Such a massive CD8 ${ }^{+}$ T-cell response is not unique to LCMV infection, as large numbers of antigen-specific $\mathrm{T}$ cells are generated in other human and animal infections ${ }^{95-99}$. In this case of a viral or bacterial pathogen in which multiple pathogen-derived epitopes are known and the specific $\mathrm{CD} 8^{+} \mathrm{T}$-cell response to each one can be quantitated by tetramer staining, the composition of the memory pool reflects the epitope specificity of the effector population ${ }^{94,100}$. The dominant epitopes in the initial response remain dominant in the memory repertoire.

The problem for the immune system is how to revert to homeostasis from a situation at the height of response when over half of the $\mathrm{CD}^{+} \mathrm{T}$ cells in the infected animal are effector cells specific for one pathogen. The system copes extremely well. During the few days after antigen has been cleared, 90-99\% of the effector cells die, leaving an expanded population of new memory cells. The original naïve $\mathrm{CD}^{+} \mathrm{T}$-cell pool is left intact, except for the promotion of the pathogen-specific members, whereas the memory pool adds some new members (Fig. 4). A recent experiment in which memory T cells were irreversibly marked by the activation of a modified granzyme $\mathrm{B}$ promoter suggests that a specific subset of effector cells contributes to memory ${ }^{101}$. What regulates the contraction of the overwhelming numbers of effector $\mathrm{CD}^{+} \mathrm{T}$ cells into reasonable numbers of memory cells is unknown. There is no evidence that Fas/FasL or any other tumour-necrosis factor family member is involved in the massive apoptosis of the effector cells ${ }^{102}$. Cessation of antigen stimulation and withdrawal of growth factors at the end of the response may lead to death. By whatever mechanism the immune system pulls it off, this represents a remarkable example of the recovery of homeostasis in the T-cell repertoire.

\section{The lives of a $T$ cell}

$\mathrm{CD}^{+}$and $\mathrm{CD}^{+} \mathrm{T}$ cells depend on TCR interactions with selfpeptides presented by MHC molecules for both their maturation in the thymus and their maintenance in peripheral lymphoid organs. The signals delivered in the periphery through interactions with self maintain the homeostasis of the naïve T-cell pool by keeping cells alive and by stimulating a low level of proliferation when the need arises. Low-affinity interactions with self-peptide-MHC may keep naïve $\mathrm{T}$ cells alert and is a way for them to reaffirm their potential to respond to higher affinity interactions with foreign peptide. The recognition of self-peptide-MHC by developing thymocytes does more than simply deliver a survival signal-it also allows the preferential selection of $\mathrm{T}$ cells on the basis of their ability to detect self-ligands with high specificity and low affinity. Because the composition of self-peptides bound to MHC is diverse, the specificity of $\mathrm{T}$ cells selected to mature is also diverse. The fact that peripheral $\mathrm{T}$ cells remain sensitive to self-peptide-MHC suggests that a continuum of self-recognition ensures diversity within the Tcell pool long after positive selection in the thymus is completed.

The factors that govern the production and maintenance of the memory T-cell pool are a mystery. This issue is further compounded by the finding that memory $\mathrm{T}$ cells are heterogeneous and can be subclassified into those that retain receptors for entering secondary lymphoid organs and those that preferentially enter inflamed tissues and perform immediate effector function ${ }^{103}$. In contrast to naïve $\mathrm{T}$ cells, T cells that have responded to foreign antigen and graduated to the memory pool no longer require self-antigen stimulation to survive. An obvious rational for this is that once a $\mathrm{T}$ cell has responded to a first encounter with a pathogen, it does not need to reaffirm its usefulness in the memory pool. A pathogen that came once may certainly come again. Another reason why memory T cells should be less responsive to self-antigen is that they are potentially more dangerous than naïve cells. Memory $\mathrm{T}$ cells are easier to trigger, faster to respond, and may enter tissues more readily than naïve $\mathrm{T}$ cells, allowing them to see a broader range of self-antigens. Thus, compared with naïve $\mathrm{T}$ cells, they pose an increased threat to upset the balance of discrimination between self and non-self. On the basis of this realization, one may propose a way in which a fraction of effector cells from a primary response is allowed to revert to memory status. The effector cells that have responded well to foreign antigen, yet are the least sensitive to low-affinity selfpeptide-MHC interactions, may be the ones chosen to enter the memory pool. Their lower sensitivity to self-antigen would guard against a harmful autoimmune response due to unforeseen crossreactivity with self. This lowered sensitivity to self may be based on the specificity of their TCR in a polyclonal population, or on some stochastic change in their ability to perceive low-affinity ligands in a monoclonal setting.

The goal of vaccination is to induce long-lived memory cells. Understanding the developmental progression of $\mathrm{T}$ lymphocytes from the thymus, to naïve peripheral cell, to effector cell to memory cell is a challenge for the future. Advances in the way that we are able to identify $\mathrm{T}$ cells on the basis of their specificity, and expanding knowledge of signalling pathways will allow rapid progress in this area.

1. Zinkernagel, R. M. \& Doherty, P. C. Restriction of in vitro T cell-mediated cytotoxicity in lymphocytic choriomeningitis within a syngeneic or semiallogeneic system. Nature 248, 701-710 (1974).

2. Babbitt, B. P., Allen, P. M., Matsueda, G., Haber, E. \& Unanue, E. R. Binding of immunogenic peptides to Ia histocompatibility molecules. Nature 317, 359-361 (1985).

3. Townsend, A. R., Gotch, F. M. \& Davey, J. Cytotoxic T cells recognize fragments of the influenza nucleoprotein. Cell 42, 457-467 (1985).

4. Shortman, K. \& Wu, L. Early T lymphocyte progenitors. Annu. Rev. Immunol. 14, $29-47$ (1996).

5. Jameson, S. C., Hogquist, K. A. \& Bevan, M. J. Positive selection of thymocytes. Annu. Rev. Immunol. 13, 93-126 (1995).

6. Kisielow, P. \& von Boehmer, H. Development and selection of T cells: facts and puzzles. $A d v$. Immunol. 58, 87-209 (1995).

7. Davis, M. M. \& Bjorkman, P. J. T-cell antigen receptor genes and T-cell recognition. Nature 334, 395-402 (1988).

8. Bjorkman, P. J. et al. Structure of the human class I histocompatibility antigen, HLA-A2. Nature 329, 506-512 (1987).

9. Brown, J. H. et al. Three-dimensional structure of the human class II histocompatibility antigen HLA-DR1. Nature 364, 33-39 (1993).

10. Scott, C. A., Peterson, P. A., Teyton, L. \& Wilson, I. A. Crystal structures of two I-Ad-peptide complexes reveal that high affinity can be achieved without large anchor residues. Immunity 8, 319329 (1998).

11. Garcia, K. C. et al. An alpha beta T cell receptor structure at $2.5 \AA$ and its orientation in the TCRMHC complex. Science 274, 209-219 (1996).

12. Garcia, K. C. et al. Structural basis of plasticity in T cell receptor recognition of a self peptide-MHC antigen. Science 279, 1166-1172 (1998).

13. Garboczi, D. N. et al. Structure of the complex between human T-cell receptor, viral peptide and HLA-A2. Nature 384, 134-141 (1996).

14. Ding, Y. H. et al. Two human T cell receptors bind in a similar diagonal mode to the HLA-A2/Tax peptide complex using different TCR amino acids. Immunity 8, 403-411 (1998).

15. Garcia, K. C., Teyton, L. \& Wilson, I. A. Structural basis of T cell recognition. Annu. Rev. Immunol. 17, 369-397 (1999).

16. Teng, M. K. et al. Identification of a common docking topology with substantial variation among different TCR-peptide-MHC complexes. Curr. Biol. 8, 409-412 (1998).

17. Fehling, H. J. \& von Boehmer, H. Early alpha beta T cell development in the thymus of normal and genetically altered mice. Curr. Opin. Immunol. 9, 263-275 (1997).

18. Irving, B. A., Alt, F. W. \& Killeen, N. Thymocyte development in the absence of pre-T cell receptor extracellular immunoglobulin domains. Science 280, 905-908 (1998).

19. Zerrahn, J., Held, W. \& Raulet, D. H. The MHC reactivity of the T cell repertoire prior to positive and negative selection. Cell 88, 627-636 (1997).

20. Merkenschlager, M. et al. How many thymocytes audition for selection? J. Exp. Med. 186, 1149-1158 (1997).

21. Sim, B. C., Zerva, L., Greene, M. I. \& Gascoigne, N. R. J. Control of MHC restriction by TCR Valpha CDR1 and CDR2. Science 273, 963-966 (1996).

22. Sim, B. C. et al. Thymic skewing of the CD4/CD8 ratio maps with the T-cell receptor alphachain locus. Curr. Biol. 8, 701-704 (1998).

23. van Meerwijk, J. P. M. et al. Quantitative impact of thymic clonal deletion on the T cell repertoire. J. Exp. Med. 185, 377-383 (1997).

24. Robey, E. \& Fowlkes, B. J. Selective events in T cell development. Annu. Rev. Immunol. 12, 675-705 (1994).

25. Nikolic-Zugic, J. \& Bevan, M. J. Role of self-peptides in positively selecting the T-cell repertoire. Nature 344, 65-67 (1990).

26. Sha, W. C. et al. Positive selection of transgenic receptor-bearing thymocytes by $\mathrm{Kb}$ antigen is altered by Kb mutations that involve peptide binding. Proc. Natl Acad. Sci. 87, 6186-6190 (1990).

27. Hogquist, K. A., Gavin, M. A. \& Bevan, M. J. Positive selection of $\mathrm{CD} 8^{+} \mathrm{T}$ cells induced by major histocompatibility complex binding peptides in fetal thymic organ culture. J. Exp. Med. 177, 14691473 (1993).

28. Ashton-Rickardt, P. G., Van Kaer, L., Schumacher, T. N., Ploegh, H. L. \& Tonegawa, S. Peptide contributes to the specificity of positive selection of $\mathrm{CD}^{+} \mathrm{T}$ cells in the thymus. Cell 73, 1041-1049 (1993).

29. Ashton-Rickardt, P. G. et al. Evidence for a differential avidity model of T cell selection in the thymus. Cell 76, 651-663 (1994). 
30. Hogquist, K. A. et al. T cell receptor antagonist peptides induce positive selection. Cell 76, 17-27 (1994).

31. Sebzda, E. et al. Mature $\mathrm{T}$ cell reactivity altered by peptide agonist that induces positive selection. $J$. Exp. Med. 183, 1093-1104 (1996).

32. Hogquist, K. A., Jameson, S. C. \& Bevan, M. J. Strong agonist ligands for the T cell receptor do not mediate positive selection of functional $\mathrm{CD}^{+} \mathrm{T}$ cells. Immunity 3, 79-86 (1995).

33. Davis, M. M. et al. Ligand recognition by alpha beta T cell receptors. Annu. Rev. Immunol. 16, 523 544 (1998).

34. Alam, S. M. et al. T-cell-receptor affinity and thymocyte positive selection. Nature 381, 558-559 (1996).

35. Hogquist, K. A. et al. Specific recognition of thymic self-peptides induces the positive selection of cytotoxic T lymphocytes. Immunity 7, 221-231 (1997).

36. Hu, Q. et al. Specific recognition of thymic self-peptides induces the positive selection of cytotoxic $\mathrm{T}$ lymphocytes. Immunity 7, 221-231 (1997).

37. Baldwin, K. K., Reay, P. A., Wu, L., Farr, A. \& Davis, M. M. A T cell receptor-specific blockade of positive selection. J. Exp. Med. 189, 13-24 (1999).

38. Kersh, G. J. \& Allen, P. M. Essential flexibility in the T-cell recognition of antigen. Nature 380, 495498 (1996).

39. Fung-Leung, W. P. et al. Antigen presentation and T cell development in H2-M-deficient mice. Science 271, 1278-1281 (1996).

40. Martin, W. D. et al. H2-M mutant mice are defective in the peptide loading of class II molecules, antigen presentation, and T cell repertoire selection. Cell 84, 543-550 (1996).

41. Miyazaki, T. et al. Mice lacking H2-M complexes, enigmatic elements of the MHC class II peptideloading pathway. Cell 84, 531-541 (1996).

42. Ignatowicz, L., Kappler, J. \& Marrack, P. The repertoire of T cells shaped by a single MHC/peptide ligand. Cell 84, 521-541 (1996).

43. Fukui, Y. et al. Positive and negative $\mathrm{CD}^{+}$thymocyte selection by a single MHC class II/peptide ligand affected by its expression level in the thymus. Immunity 6, 401-410 (1997).

44. Grubin, C. E., Kovats, S., deRoos, P. \& Rudensky, A. Y. Deficient positive selection of CD4 T cells in mice displaying altered repertoires of MHC class II-bound self-peptides. Immunity 7, 197-208 (1997).

45. Tourne, S. et al. Selection of a broad repertoire of $\mathrm{CD}^{+} \mathrm{T}$ cells in $\mathrm{H}-2 \mathrm{Ma} / 0$ mice. Immunity $7,187-$ 195 (1997).

46. Surh, C. D., Lee, D. S., Fung-Leung, W. P., Karlsson, L. \& Sprent, J. Thymic selection by a single $\mathrm{MHC} /$ peptide ligand produces a semidiverse repertoire of $\mathrm{CD}^{+} \mathrm{T}$ cells. Immunity 7, 209-219 (1997).

47. Sant'Angelo, D. B. et al. The imprint of intrathymic self-peptides on the mature T cell receptor repertoire. Immunity 7, 517-524 (1997).

48. Barton, G. M. \& Rudensky, A. Y. Requirement for diverse, low-abundance peptides in positive selection of T cells. Science 283, 67-70 (1999).

49. Davey, G. M. et al. Preselection thymocytes are most sensitive to T cell receptor stimulation than mature T cells. J. Exp. Med. 188, 1867-1874 (1998).

50. Lucas, B., Stefanova, I., Yasutomo, K., Dautigny, N. \& Germain, R. N. Divergent changes in the sensitivity of maturing $\mathrm{T}$ cells to structurally related ligands underlies formation of a useful $\mathrm{T}$ cel repertoire. Immunity 10, 367-376 (1999).

51. Tanchot, C., Rosado, M. M., Agenes, F., Freitas, A. A. \& Rocha, B. Lymphocyte homeostasis. Semin Immunol. 9, 331-337 (1997).

52. Mombaerts, P. et al. Mutations in T-cell antigen receptor genes alpha and beta block thymocyte development at different stages. Nature 360, 225-231 (1992).

53. Kitamura, D., Roes, J., Kuhn, R. \& Rajewsky, K. A B-cell deficient mouse by targeted disruption of the membrane exon of the immunoglobulin mu chain gene. Nature 350, 423-426 (1991).

54. Bender, J., Mitchell, T., Kappler, J. \& Marrack, P. $\mathrm{CD}^{+}{ }^{+} \mathrm{T}$ cell division in irradiated mice requires peptides distinct from those responsible for thymic selection. J. Exp. Med. 190, 367-374 (1999).

55. Tanchot, C. \& Rocha, B. The peripheral T cell repertoire: independent homeostatic regulation of virgin and activated CD8 ${ }^{+} \mathrm{T}$ cell pools. Eur. J. Immunol. 25, 2127-2136 (1995).

56. Rocha, B., Dautigny, N. \& Pereira, P. Peripheral T lymphocytes: expansion potential and homeostatic regulation of pool sizes and CD4/CD8 ratios in vivo. Eur. J. Immunol. 19, 905-911 (1989).

57. Ernst, B., Lee, D. Chang, J. M., Sprent, J. \& Surh, C. D. The peptide ligands mediating positive selection in the thymus control $\mathrm{T}$ cell survival and homeostatic proliferation in the periphery. Immunity 11, 173-181 (1999).

58. Berzins, S. P., Boyd, R. L. \& Miller, J. F. A. P. The role of the thymus and recent thymic migrants in the maintenance of the adult peripheral lymphocyte pool. J. Exp. Med. 187, 1839-1848 (1998).

59. Tanchot, C. \& Rocha, B. Peripheral selection of T cell repertoires: the role of continuous thymus output. J. Exp. Med. 186, 1099-1106 (1997).

60. Bell, E. B., Sparshott, S. M., Drayson, M. T. \& Ford, W. L. The stable and permanent expansion of functional $\mathrm{T}$ lymphocytes in athymic nude rats after a single injection of mature T cells. J. Immunol. 139, 1379-1384 (1987).

61. Sprent, J. Lifespans of naive, memory and effector lymphocytes. Curr. Opin. Immunol. 5, 433-438 (1993).

62. Sprent, J., Schaefer, M., Hurd, M., Surh, C. D. \& Ron, Y. Mature murine B and T cells transferred to SCID mice can survive indefinitely and many maintain a virgin phenotype. J. Exp. Med. 174, 717728 (1991).

63. Tough, D. F \& Sprent, J. Turnover of naive- and memory-phenotype T cells. J. Exp. Med. 179, 1127 1135 (1994).

64. von Boehmer, H. \& Hafen, K. The life span of naive $\alpha / \beta$ T cells in secondary lymphoid organs. J. Exp. Med. 177, 891-896 (1993).

65. Bruno, L., von Boehmer, H. \& Kirberg, J. Cell division in the compartment of naive and memory T lymphocytes. Eur. J. Immunol. 26, 3179-3184 (1996).

66. Kuo, C. T., Veselitis, M. L. \& Leiden, J. M. LKLF: a transcriptional regulator of single-positive T cell quiescence and survival. Science 277, 1986-1990 (1997).

67. Oukka, M. et al. The transcription factor NFAT4 is involved in the generation and survival of T cells. Immunity 9, 295-304 (1998).
68. Lodolce, J. P. et al. IL-15 receptor maintains lymphoid homeostasis by supporting lymphocyte homing and proliferation. Immunity 9, 669-676 (1998).

69. Nakajima, H., Shores, E. W., Noguchi, M. \& Leonard, W. J. The common cytokine receptor $\gamma$ chain plays an essential role in regulating lymphoid homeostasis. J. Exp. Med. 185, 189-195 (1997).

70. Veis, D. J., Sorenson, C. M., Shutter, J. R. \& Korsmeyer, S. J. Bcl-2-deficient mice demonstrate fulminant lymphoid apoptosis, polycystic kidneys, and hypopigmented hair. Cell 75, 229-240 (1993).

71. Freitas, A. A. \& Rocha, B. Peripheral T cell survival. Curr. Opin. Immunol. 11, 152-156 (1999).

72. Kirber, J., Berns, A. \& von Boehmer, H. Peripheral T cell survival requires continual ligation of the $\mathrm{T}$ cell receptor to major histocompatibility complex-encoded molecules. J. Exp. Med. 186, 1269-1275 (1997).

73. Takeda, S., Rodewald, H.-R., Arakawa, H., Bluethmann, H. \& Shimizu, T. MHC class II molecules are not required for survival of newly generated $\mathrm{CD} 4^{+} \mathrm{T}$ cells, but affect their long-term life span. Immunity 5, 217-228 (1996).

74. Brocker, T. Survival of mature CD4 T lymphocytes is dependent on major histocompatibility complex class II-expressing dendritic cells. J. Exp. Med. 186, 1223-1232 (1997).

75. Rooke, R., Waltzinger, C., Benoist, C. \& Mathis, D. Targeted complementation of MHC class II deficiency by intrathymic delivery of recombinant adenoviruses. Immunity 7, 123-134 (1997).

76. Tanchot, C., Lemonnier, F. A., Perarnau, B., Freitas, A. A. \& Rocha, B. Differential requirements for survival and proliferation of CD8 naive or memory T cells. Science 276, 2057-2062 (1997).

77. Murali-Krishna, K. et al. Persistence of memory CD8 T cells in MHC class I deficient mice. Science (in the press).

78. Nesic, D. \& Vukmanovic, S. MHC class I is required for peripheral accumulation of $\mathrm{CD} 8^{+}$thymic emigrants. J. Immunol. 160, 3705-3712 (1998).

79. Bell, E. B., Sparshott, S. M., Drayson, M. T. \& Hunt, S. V. The origin of T cells in permanently reconstituted old athymic nude rats. Analysis using chromosome or allotype markers. Immunology 68, 547-556 (1989).

80. McDonagh, M. \& Bell, E. B. The survival and turnover of mature and immature CD8 T cells. Immunology 84, 514-520 (1995)

81. Pereira, P. \& Rocha, B. Post-thymic in vivo expansion of mature $\alpha \beta$ T cells. Int. Immunol. 3, 10771080 (1991).

82. Oehen, S. \& Brduscha-Riem, K. Naive cytotoxic T lymphocytes spontaneously acquire effector function in lymphocytopenic recipients: A pitfall for T cell memory studies? Eur. J. Immunol. 29, 608-614 (1999).

83. Sprent, J., Surh, C. D. \& Tough, D. Fate of Tand B cells transferred to SCID mice. Res. Immunol. 145, 328-331 (1994).

84. Bell, E. B. \& Sparshott, S. M. The peripheral T-cell pool: regulation by non-antigen induced proliferation? Semin. Immunol. 9, 347-353 (1997).

85. Mackall, C. L. et al. Thymic-independent T cell regeneration occurs via antigen-driven expansion of peripheral $\mathrm{T}$ cells resulting in a repertoire that is limited in diversity and prone to skewing. $J$. Immunol. 156, 4609-4616 (1996).

86. Viret, C., Wong, F. S. \& Janeway, C. S. Designing and maintaining the mature TCR repertoire: the continuum of self-peptide:self-MHC complex recognition. Immunity 10, 559-568 (1999).

87. Beutner, U. \& MacDonald, H. R. TCR-MHC class II interaction is required for peripheral expansion of CD4 cells in a T cell-deficient host. Int. Immun. 10, 305-310 (1998).

88. Kieper, W. C. \& Jameson, S. C. Homeostatic expansion and phenotypic conversion of naive T cells in response to self peptide/MHC ligands. Proc. Natl Acad. Sci. USA (in the press).

89. Goldrath, A. W. \& Bevan, M. J. Low affinity ligands for the TCR drive proliferation of mature CD8 ${ }^{+}$ T cells in lymphopenic hosts. Immunity 11, 183-190 (1999).

90. Markiewicz, M. A. et al. Long-term T cells memory requires the surface expression of self-peptide/ major histocompatibility complex molecules. Proc. Natl Acad. Sic. USA 95, 3065-3070 (1998).

91. Garcia, S., DiSanto, J. \& Stockinger, B. Following the development of a CD4 T cell immune response in vivo: from activation to memory formation. Immunity 11, 163-171 (1999).

92. Swain, S., Hui, H. \& Huston, G. Class II-independent generation of CD4 memory T cells from effectors. Science (in the press)

93. Butz, E. A. \& Bevan, M. J. Massive expansion of antigen-specific $\mathrm{CD} 8^{+} \mathrm{T}$ cells during an acute virus infection. Immunity 8, 167-175 (1998).

94. Murali-Krishna, K. et al. Counting antigen-specific CD8 T cells: a reevaluation of bystander activation during viral infection. Immunity 8, 177-187 (1998).

95. Busch, D. H., Pilip, I. M., Vijh, S. \& Pamer, E. G. Coordinate regulation of complex T cell populations responding to bacterial infection. Immunity 8, 353-362 (1998).

96. Callan, M. F. et al. Direct visualization of antigen-specific $\mathrm{CD}^{+} \mathrm{T}$ cells during the primary immune response to Epstein-Barr virus in vivo. J. Exp. Med. 187, 1395-1402 (1998).

97. Cose, S. C., Jones, C. M., Wallace, M. E., Heath, W. R. \& Carbone, F. R. Antigen-specific CD8 ${ }^{+}$T cell subset distribution in lymph nodes draining the site of herpes simplex virus infection. Eur. J. Immunol. 27, 2310-2316 (1997).

98. Flynn, K. J. et al. Virus-specific $\mathrm{CD}^{+} \mathrm{T}$ cells in primary and secondary influenza pneumonia. Immunity 8, 683-691 (1998).

99. Zimmermann, C. \& Pircher, H. A novel approach to visualize polyclonal virus-specific CD8 T cells in vivo. J. Immunol. 162, 5178-5182 (1999).

100. Busch, D. H., Pilip, I. \& Pamer, E. G. Evolution of a complex T cell receptor repertoire during primary and recall bacterial infection. J. Exp. Med. 188, 61-70 (1998).

101. Jacob, J. \& Baltimore, D. Modelling T-cell memory by genetic marking of memory T cells in vivo. Nature 399, 593-597 (1999).

102. Razvi, E. S., Jiang, Z., Woda, B. A. \& Welsh, R. M. Lymphocyte apoptosis during the silencing of the immune response to acute viral infections in normal, Ipr, and Bcl-2-transgenic mice. Am. J. Pathol. 147, 79-91 (1995).

103. Sallusto, F., Lenig, D., Förster, R., Lipp, M. \& Lanzavecchia, A. Two subsets of memory T lymphocytes with distinct homing potentials and effector functions. Nature 401, 708-712 (1999).

Correspondence should be addressed to M.J.B. (e-mail: mbevan@u.washington.edu). 